\title{
La Déclaration Universelle des Droits de l'Homme comme l'expression d'une vision du monde : une approche topique et génétique
}

The Universal Declaration of Human Rights as an expression of a "vision of the world": a topical and genetic perspective

Emmanuel de Jonge

\section{(2) OpenEdition Journals}

\section{Édition électronique}

URL : http://journals.openedition.org/aad/956

DOI : 10.4000/aad.956

ISSN : 1565-8961

\section{Éditeur}

Université de Tel-Aviv

\section{Référence électronique}

Emmanuel de Jonge, «La Déclaration Universelle des Droits de l'Homme comme l'expression d'une vision du monde : une approche topique et génétique », Argumentation et Analyse du Discours [En ligne], 4 | 2010, mis en ligne le 15 avril 2010, consulté le 09 octobre 2020. URL : http://

journals.openedition.org/aad/956; DOI : https://doi.org/10.4000/aad.956

Ce document a été généré automatiquement le 9 octobre 2020.

\section{cc) (†) $\odot$}

Argumentation \& analyse du discours est mis à disposition selon les termes de la licence Creative Commons Attribution - Pas d'Utilisation Commerciale - Pas de Modification 4.0 International. 


\section{La Déclaration Universelle des Droits de l'Homme comme l'expression d'une vision du monde : une approche topique et génétique}

The Universal Declaration of Human Rights as an expression of a "vision of the world": a topical and genetic perspective

Emmanuel de Jonge

\section{Préliminaires}

1 La réflexion que je souhaiterais développer ici est le prolongement d'une recherche que j'ai entamée ailleurs (de Jonge 2008a, 2008b). J'ai cherché à montrer que les grandes déclarations (en particulier la Déclaration d'Indépendance américaine, la Déclaration des Droits de l'Homme et du Citoyen ou la Déclaration Universelle des Droits de l'Homme) sont l'expression linguistique des fondements éthiques, politiques et philosophiques d'une société particulière à une époque donnée. Ces fondements, que l'on pourra décrire en termes de représentations collectives et de croyances partagées constituent le monde commun d'une société, c'est-à-dire ce qui fait l'identité collective de ses membres. Nous pourrons appeler l'ensemble de ces représentations la «vision du monde» de la communauté, à la suite de Jean-Marie Schaeffer, qui, dans son ouvrage La fin de l'exception humaine, en donne une définition qui associe la dimension collective du concept à une approche socio-cognitive :

Fonctionnellement, une vision du monde livre une justification de la réalité. Bien qu'elle comporte des explications causales - sur l'origine du monde, sur sa constitution, sur la provenance et le destin de l'homme, sur l'organisation sociale, etc. -, celles-ci ressemblent aux étiologies des fables: elles ont pour fonction de sauver la réalité (pour nous), au même titre que le récit d'une fable justifie un adage de prudence ou de morale. [...] Autrement dit, elle dé-problématise la réalité en la réduisant à une représentation transparente, cohérente et ultimement satisfaisante 
(pour l'être humain). Cette procédure, décrite ici en des termes abstraits, prend des formes différentes selon les époques et les cultures, mais le résultat auquel elle aboutit invariablement peut s'énoncer simplement: la vision du monde donne une signification à la vie. (2008: 364-365)

2 La vision du monde désigne ainsi à la fois une certaine idée de l'homme et une conception de la société ; elle détermine les rapports entre les individus dans une communauté et encadre le contenu de leurs représentations culturelles - ce dernier terme étant entendu au sens large, il inclut tout autant les productions intellectuelles, philosophiques et politiques, que les échanges interindividuels de la vie publique.

3 Au plan rhétorique, la vision du monde se traduit sous la forme de ce que je propose d'appeler « l'arrière-plan topique ». Il s'agit d'une strate de représentations collectives qui sont organisées et hiérarchisées dans une société donnée, et déterminent le contenu rhétorique des échanges dans cette société. Les individus iront puiser dans cet arrière-plan topique des représentations, dont l'expression sera la manifestation de l'appartenance à la même communauté. L'arrière-plan topique peut donc être défini comme une strate intermédiaire de représentations qui se situe entre l'esprit des individus (la cognition individuelle) et l'expression rhétorique de représentations collectives par le discours. Il est une interface entre l'esprit de l'individu (la cognition) et ses productions discursives au sein de la communauté.

Posons à présent que chaque communauté possède son arrière-plan topique. Les contenus de l'arrière-plan topique dépendront des fondements de la société; de comment la société se représente à la base son identité en tant que groupe, de son histoire collective. Pour le dire en d'autres termes, il dépendra de la nature du lien entre les membres de la communauté, de la raison pour laquelle un ensemble d'individus s'est rassemblé et a décidé de s'associer en communauté. La rhétorique d'une communauté sera alors façonnée à l'image de cet arrière-plan topique fondationnel, dont elle se nourrira. Les déclarations expriment selon moi cet arrièreplan topique fondationnel. Elles sont, pour le dire en termes aristotéliciens, un réservoir de lieux propres et lieux communs, lesquels formeront les fondements topiques qui seront mobilisés dans les discours et les échanges en société. Métaphoriquement, on pourrait dire qu'elles sont le patrimoine topique des sociétés dans lesquelles elles sont proclamées. Cela signifie qu'en les analysant linguistiquement, on peut décrire les fondements de la rhétorique d'une société. C'est le travail que je me propose d'effectuer dans cette contribution.

5 Je m'intéresserai à la Déclaration Universelle des Droits de l'Homme (que j'abrégerai désormais par DUDH). Mon hypothèse est que la DUDH est l'expression d'un arrièreplan topique, celui du monde occidental de l'après-guerre, et plus particulièrement de l'Europe occidentale traumatisée par son histoire récente et les horreurs nazies. Les droits de l'homme sont en ce sens le cadre dans lequel prendront place les représentations collectives des peuples occidentaux, incarnant ainsi une façon de penser le monde et l'homme, de se définir par rapport aux autres, d'élaborer des attentes et des croyances au niveau social. La notion de droits de l'homme telle qu'elle est conçue dans cette vision du monde recouvre une réalité nouvelle. A la différence de la conception des droits de l'homme exprimée dans la Déclaration de 1789, lesquels s'articulaient autour de la recherche de l'égalité politique entre les hommes, la ligne directrice de l'arrière-plan topique propre à la DUDH est le rejet de l'idéologie nazie et la recherche de moyens de protections contre celle-ci. A cet égard, je qualifierai les droits de l'homme tels qu'ils sont conçus en 1948 de «miroir inversé du nazisme ». En 
effet, dans leurs travaux, les rédacteurs ont systématiquement cherché à élaborer des principes en réaction à, voire en opposition avec ceux du régime nazi. L'interdiction de la torture, le principe de non-discrimination, le droit à la dignité sont autant de droits qui sont issus de l'ambition des rédacteurs, laquelle était de protéger les peuples du retour potentiel du nazisme. La DUDH a pour arrière-plan le rejet radical des actes barbares et la volonté que ceux-ci ne se reproduisent plus jamais (voir aussi Morsink 2000).

\section{Contexte historique}

Proclamée au sortir de la guerre, en 1948, la DUDH est le fruit d'un travail de réflexion qui dura près de deux ans. L'idée provenait de Franklin Roosevelt, qui avait l'ambition d'écrire après la guerre une grande charte adressée aux peuples du monde. Après son décès en 1945, sa femme, Eleanor Roosevelt, prit le relais et mit en place une réflexion sur les Droits de l'Homme au sein des Nations Unies. Plusieurs commissions furent créées, et l'idée d'une déclaration vit progressivement le jour. Initialement, le but de la Commission des Droits de l'Homme était de produire un document qui aurait un statut de convention pour contraindre les nations à se conformer aux principes des droits de l'homme. Très vite, les rédacteurs de la future charte se rendirent compte de la difficulté d'une telle entreprise, en même temps qu'émergeait le désir de rédiger une charte qui, en plus de s'adresser aux nations, pourrait s'adresser aux peuples et aux individus. Ils divisèrent leurs travaux en deux parties, l'une consacrée à la rédaction d'une convention avec force normative, l'autre consacrée à la rédaction d'une déclaration avec force morale et devant servir d'instrument pédagogique. Le Comité de Rédaction de la Déclaration des Droits de l'Homme fut créé et commença ses travaux en 1947. Pour dire le vrai, à la lecture des comptes-rendus de rédaction, on a le sentiment que personne ne pouvait vraiment définir le rôle que serait amenée à remplir la future déclaration, et pourtant tous les membres de la Commission des Droits de l'Homme avaient bien l'intuition qu'il était nécessaire d'en rédiger une. A l'issue de trois sessions de discussions (chacune d'une quinzaine de jours environ), au cours desquelles les rédacteurs, en plus de leurs observations personnelles, considéraient les propositions faites par les Etats, une version provisoire fut soumise à l'Assemblée Générale des Nations Unies. L'Assemblée prit environ deux mois (d'octobre à début décembre 1948) pour discuter et amender le projet, lequel fut proclamé par les Etats signataires le 10 décembre 1948.

7 Ce qui est remarquable dans ce processus de rédaction - et qui constitue une véritable mine pour le linguiste - est le fait qu'au fil des travaux, se construit progressivement une vision du monde qui vient exprimer un sentiment nouveau, celui de l'émotion provoquée par le spectacle de la catastrophe de 1940-45. Progressivement, tout se passe comme si les rédacteurs étaient guidés par une conviction profonde selon laquelle l'ancienne vision du monde s'effondrait peu à peu. Ils s'attachent alors à élaborer une déclaration qui reflétait un nouvel arrière-plan topique universaliste, résolument contre la guerre, compatissante envers les victimes et cherchant à dépasser le traumatisme de la Deuxième Guerre Mondiale. Ainsi, tous les éléments, dans les versions préliminaires de la Charte, qui exprimaient des reliquats de l'ancien paradigme des droits de l'homme furent progressivement supprimés, pour faire place à l'expression d'une vision du monde renouvelée. Ainsi, la première version de la charte 
définissait les règles que les individus devaient respecter vis-à-vis de leur Etats, et suivait ainsi l'ancienne conception des rapports politiques entre Etat et individus, où l'individu était avant tout subordonné à l'Etat. Mais suite aux constatations établies concernant les responsabilités des Etats dans les actes perpétrés contre les individus pendant la Deuxième Guerre Mondiale, le modèle fut complètement renversé et la DUDH devint une déclaration centrée sur les droits des individus et les règles que devaient respecter les Etats à leur égard. Le changement de l'adjectif « international ", initialement choisi pour le titre de la déclaration, en « universel » est une manifestation de ce changement de perspective ${ }^{1}$.

\section{Les valeurs comme expression rhétorique de la vision du monde}

De par leur statut fondateur, les déclarations doivent remplir deux fonctions. On dira que la déclaration doit en même temps poser un cadre fondateur pour la vision du monde d'une société donnée et faire adhérer ses membres à cette vision du monde. D'un côté, poser le cadre fondateur se traduit par l'énumération d'une liste de valeurs et de principes qui expriment le nouvel arrière-plan topique. Le contenu axiologique et juridique de la déclaration est alors présenté sous la forme de propositions générales et se voit conférer le statut de vérité indiscutable et anhistorique. C'est là d'ailleurs une caractéristique commune à toutes les déclarations dont on peut comprendre très facilement les raisons. D'une part, si les valeurs étaient présentées comme des décisions prises en contexte ou des jugements ad hoc, elles ne pourraient constituer un fondement stable sur lequel la société peut construire ses croyances et développer sa vision du monde. D'autre part, susciter l'adhésion autour d'une vision du monde doit se faire en permettant aux individus de se reconnaître et de s'identifier à la communauté via des mécanismes d'évocation, comme le rappel, par un récit, d'une mémoire collective ${ }^{2}$. Ce processus permet également d'incarner les valeurs en les identifiant aux raisons historiques qui ont conduit à leur adoption par la communauté. Mais ce processus d'incarnation pour permettre l'identification rend par contrecoup les valeurs moins abstraites et donc moins générales.

9 Nous verrons donc qu'il existe, dans les déclarations, deux formes d'expression linguistiques des valeurs. Elles s'expriment soit sous la forme de propositions générales, soit elles s'inscrivent dans un contexte spécifique, relatif à l'histoire récente de la communauté. Ces deux formes d'expression correspondent aux deux fonctions de la déclaration : poser un cadre fondamental comme inaliénable et indiscutable et faire adhérer les membres de la communauté à celui-ci. La première est une fonction d'ordre socio-anthropologique, la seconde est une fonction rhétorique et même épidictique. Au plan strictement rhétorique, la déclaration cristallise à elle seule deux ambitions: forger les contours de ce qui deviendra le genre délibératif, en fixant les lieux de la cité nouvellement créée, et constituer un objet de communion épidictique originel pour les membres de la Cité.

10 Mon analyse aura donc pour but de détailler deux points complémentaires. Le premier a trait à l'expression de l'arrière-plan topique dans la DUDH par les valeurs qu'elle promeut. Je chercherai à décrire, par l'analyse du texte de la DUDH et de sa genèse, que les énoncés reflètent un arrière-plan topique particulier et ont été élaborés sur ce critère, qui est, comme on l'a dit plus haut, la recherche de moyens efficaces pour que 
les actes barbares perpétrés pendant la seconde guerre mondiale ne se reproduisent jamais plus. Dans un second temps, j'analyserai les deux formes d'expression des valeurs, proposition générale et valeurs exprimées dans un contexte historique spécifique pour montrer qu'elles sont l'instanciation d'un même arrière-plan topique, lequel est déterminé par le contexte historique dans lequel la déclaration est proclamée. Dans le cas de la DUDH, la tension entre les valeurs universelles et les valeurs ancrées historiquement est encore accentuée, puisque cette déclaration doit s'adresser à tous les peuples du monde, à la fois à ceux qui ont connu la guerre en tant que victimes, à ceux qui l'ont gagnée, mais encore à ceux qui n'ont pas vécu la guerre voire ne connaissent pas même l'idée de droits de l'homme.

11 L'analyse portera sur un corpus court : les deux premiers paragraphes du préambule de la DUDH. Le préambule est le lieu même de l'exposition des raisons qui mènent à la proclamation: soit on justifie par l'histoire récente, en rappelant les événements qui ont précédé la proclamation, soit on justifie par des principes généraux transhistoriques qui donnent du sens à l'acte de proclamation. Comme on le verra, le premier paragraphe exprime en effet les valeurs sous la forme d'une proposition générale, alors que le second les exprime plutôt sous la forme d'une proposition particulière et contextualisée. On examinera d'abord chaque signification séparément, pour se concentrer ensuite sur leur articulation.

\section{Le préambule}

12 La version finale du préambule de la DUDH se compose de sept paragraphes introduits par «considérant » et d'un paragraphe de proclamation. Les sept «considérant» forment ensemble la justification de la proclamation. Lisons le préambule de la DUDH tel qu'il fut finalement inclus à la déclaration :

Considérant que la reconnaissance de la dignité inhérente à tous les membres de la famille humaine et de leurs droits égaux et inaliénables constitue le fondement de la liberté, de la justice et de la paix dans le monde.

Considérant que la méconnaissance et le mépris des droits de l'homme ont conduit à des actes de barbarie qui révoltent la conscience de l'humanité et que l'avènement d'un monde où les êtres humains seront libres de parler et de croire, libérés de la terreur et de la misère, a été proclamé comme la plus haute aspiration de l'homme. Considérant qu'il est essentiel que les droits de l'homme soient protégés par un régime de droit pour que l'homme ne soit pas contraint, en suprême recours, à la révolte contre la tyrannie et l'oppression.

Considérant qu'il est essentiel d'encourager le développement de relations amicales entre nations.

Considérant que dans la Charte les peuples des Nations Unies ont proclamé à nouveau leur foi dans les droits fondamentaux de l'homme, dans la dignité et la valeur de la personne humaine, dans l'égalité des droits des hommes et des femmes, et qu'ils se sont déclarés résolus à favoriser le progrès social et à instaurer de meilleures conditions de vie dans une liberté plus grande.

Considérant que les Etats Membres se sont engagés à assurer, en coopération avec l'Organisation des Nations Unies, le respect universel et effectif des droits de l'homme et des libertés fondamentales.

Considérant qu'une conception commune de ces droits et libertés est de la plus haute importance pour remplir pleinement cet engagement.

L'Assemblée Générale proclame la présente Déclaration universelle des droits de l'homme comme l'idéal commun à atteindre par tous les peuples et toutes les nations 
afin que tous les individus et tous les organes de la société, ayant cette Déclaration constamment à l'esprit, s'efforcent, par l'enseignement et l'éducation, de développer le respect de ces droits et libertés et d'en assurer, par des mesures progressives d'ordre national et international, la reconnaissance et l'application universelles et effectives, tant parmi les populations des Etats Membres eux-mêmes que parmi celles des territoires placés sous leur juridiction.

\section{1. Analyse du premier alinéa}

Considérant que la reconnaissance de la dignité inhérente à tous les membres de la famille humaine et de leurs droits égaux et inaliénables constitue le fondement de la liberté, de la justice et de la paix dans le monde.

Le premier alinéa du préambule se présente comme une vérité transhistorique générale. Il a été proposé par la délégation des Etats-Unis et n'a pratiquement pas été modifié après-coup. L'énoncé exprime la croyance fondamentale selon laquelle les hommes ont des droits inaliénables, et que c'est la reconnaissance de ceux-ci qui confère à tous, disons, le «droit d'avoir des droits». La notion d'inaliénabilité des droits, que l'on retrouvait dans la Déclaration d'Indépendance américaine sous la forme de «droits sacrés » et dans la Déclaration de 1789 comme « droits naturels » répond à l'exigence de fonder les droits à l'extérieur d'eux-mêmes, c'est-à-dire qu'on doit pouvoir justifier la légitimité de la création d'une réalité sociale en dehors de cette réalité. On doit nécessairement faire reposer la réalité sociale sur un fondement extérieur à celle-ci sinon, d'un point de vue argumentatif, on s'exposerait au risque de la régression à l'infini de la justification, rendant inopérante toute justification. Comme l'affirme Angenot :

Dans la pensée post-religieuse, l'homme est son propre fondement pour penser l'institution de la société. Mais puisque j'ai besoin d'un fondement, ce ne saurait être l'homme empirique qui me le fournira, mais une «nature humaine» dont on tirera sans peine la « loi naturelle ». (2008: 175)

15 Au plan anthropologique, l'affirmation de l'inaliénabilité des droits permet de faire le lien entre le naturel et le social : elle fonde ainsi la loi dans un élément considéré comme naturel et fondamental par la société concernée (Dieu ou la nature elle-même remplissent le plus souvent ce rôle). Par exemple, la délégation des Pays-Bas a voulu amender le préambule en faisant ajouter après l'expression "les droits égaux et inaliénables", l'énoncé "fondés sur l'origine divine et la destinée immortelle de l'homme $»^{3}$. Deux pays, la Nouvelle-Zélande et l'Equateur, ont quant à eux proposé de fonder les droits de l'homme sur la «foi » dans les droits fondamentaux des êtres humains ${ }^{4}$. Soulignons ici l'intérêt de cette proposition pour l'analyste. Si elle avait été acceptée, le fondement aurait eu un tout autre statut: la «foi»dans les droits de l'homme implique que l'on « croit» aux droits de l'homme comme on croit en Dieu. Le fondement des droits de l'homme aurait alors été tributaire d'une attitude de l'individu envers ceux-ci. On pourrait y croire ou ne pas y croire, et dans ce dernier cas, les droits

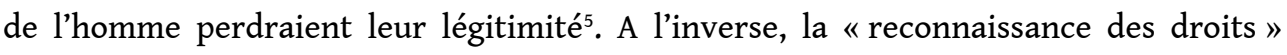
présuppose que ces droits existent indépendamment de leur reconnaissance, mais que c'est seulement en les reconnaissant qu'on les garantit. Cette version donne des bases beaucoup plus solides aux fondements de la déclaration. 

chal dans l'arrière-plan topique qu'exprime la DUDH, en témoigne sa première place dans l'alinéa 1 du préambule, comme dans l'article $1^{10}$. Or, lorsqu'on s'intéresse aux synthèses du Comité de Rédaction, on s'aperçoit que la notion de dignité est très peu commentée. On peut supposer que pour les rédacteurs, ce qu'elle recouvre relève de l'évidence, puisque lorsqu'elle est évoquée, elle ne soulève pas de discussions ni de 
débats quant à sa définition ou son domaine d'application. Alors que tous les autres principes ont fait l'objet d'intenses discussions, celui de "dignité » semble avoir fait consensus avant même le début des débats. Dès la toute première séance du Comité de Rédaction, il était supposé être partagé par l'ensemble des membres. La genèse de l'inclusion du terme de dignité est assez difficile à établir. On sait que le maréchal Smuts, membre sud-africain de la Délégation des Nations-Unies, proposa de l'inclure une première fois à la Charte des Nations Unies en 1945, dont il rédigea le préambule. Ensuite, il fut directement repris par les rédacteurs de la DUDH. La notion de dignité devait être tout à fait claire à l'esprit des rédacteurs. Il s'agissait de proposer une valeur qui permette de contrer la possibilité d'un retour des atrocités qui avaient justement atteint et détruit la dignité de l'homme. Le terme qui fut d'ailleurs utilisé à l'origine, dans la première version du préambule de la Charte des Nations Unies en 1945, était celui d'« intégrité » qui évoquait a contrario, plus directement encore que celui de "dignité », le lien avec les actes commis par les Nazis. Dans les comptes-rendus de la DUDH, on trouve une confirmation assez forte de cette intuition. Ainsi, dans l'article 8 d'une première version de la Déclaration en anglais, on trouve l'expression suivante : "No one shall be subjected to torture, or to cruel or inhuman punishment or indignity ${ }^{11}$. Associer l'inverse de la dignité, l'« indignité ", à la torture montre bien que dans l'esprit des rédacteurs, la notion de « dignité » n'avait rien d'abstrait. Elle a été au contraire absolutisée en miroir inversé de la représentation des violations de celle-ci pendant la Deuxième Guerre Mondiale. Ajoutons à cela le témoignage suivant, issu des premiers débats des comptes-rendus de rédaction :

M. Malik déclare que les membres du Comité de rédaction continueront à discuter dans l'abstrait s'ils ne gardent pas présents à l'esprit les causes historiques qui expliquent l'intérêt actuellement porté à la question des droits de l'homme. Ces dernières années, des hommes ont surgi, qui incarnaient des pires instincts de la nature humaine, et ont foulé aux pieds la dignité de l'homme. C'est pour cette raison que l'on désire actuellement s'assurer que de telles atrocités ne se reproduiront pas $^{12}$.

21 Cet extrait du débat exprime bien la tension entre d'une part, la recherche d'une conception des valeurs qui soient assez générales et abstraite pour toucher l'ensemble des hommes mais qui soit assez incarnée dans l'histoire récente, pour susciter la mobilisation et emporter l'adhésion des individus.

\subsubsection{La notion de famille humaine}

22 On trouve un deuxième fragment dans l'alinéa 1 qui illustre l'arrière-plan topique exprimé par la DUDH : l'expression «famille humaine ». Celle-ci fait également écho à l'article 1 de la déclaration selon lequel «Tous les êtres humains naissent libres et égaux en dignité et en droits. Ils sont doués de raison et de conscience et doivent agir les uns envers les autres dans un esprit de fraternité ». La métaphore de la famille humaine répond là aussi à sa destruction récente par les Nazis. Elle traverse d'ailleurs l'ensemble des travaux des rédacteurs. L'article 1, dans sa première version, insistait sur cette notion: "Tous les hommes sont frères. Comme êtres doués de raison et membres d'une seule famille, ils sont libres et sont égaux en dignité et en droits ${ }^{13}$. L'idée d'une fraternité humaine s'oppose au « fratricide » récent, comme l'avait qualifié le maréchal Smuts dans la première version de la Charte des Nations Unies ${ }^{14}$. La représentation de l'humanité soit comme victime de la guerre, soit comme empathique à l'égard de celle-ci, est fondamentale dans l'arrière-plan topique d'après la Deuxième 
Guerre Mondiale et sera à nouveau utilisée, comme nous le verrons, dans l'alinéa 2 du préambule. La métaphore de la famille déterminera la vision de l'homme dans son rapport à la société

L'alinéa 1, comme on a pu le voir, ajoute donc à l'idée de droits inaliénables, commune à toutes les déclarations, deux concepts qui sont spécifiques à la vision du monde exprimée par la DUDH : la dignité et la famille humaine. Ces deux concepts fondent, par ce qu'ils évoquent, l'idée qu'on se fait des droits inaliénables.

\subsection{Analyse du deuxième alinéa}

Considérant que la méconnaissance et le mépris des droits de l'homme ont conduit à des actes de barbarie qui révoltent la conscience de l'humanité et que l'avènement d'un monde où les êtres humains seront libres de parler et de croire, libérés de la terreur et de la misère, a été proclamé comme la plus haute aspiration de l'homme.

Cet énoncé est une proposition particulière qui fait référence à l'histoire récente et inscrit les valeurs dans cette histoire. Les valeurs sont associées à des représentations collectives vécues par la communauté. Dans une contribution antérieure, j'avais montré par l'analyse des aspects linguistiques comment on pouvait conclure qu'il s'agissait de références aux événements récents (de Jonge: 2008). Je résume ici brièvement ces premières observations avant de passer à une analyse plus profonde. Le passé composé du verbe principal des deux énoncés montre la proximité entre les événements dont on parle et le moment présent. À ce titre, en anglais, la forme verbale utilisée est un present perfect, ce qui renforce l'hypothèse puisque le present perfect, plus que le passé composé en français, exige pratiquement toujours un lien causal entre un passé proche et le présent ${ }^{15}$. Le premier énoncé parle des actes barbares récents (nous déterminerons lesquels ci-dessous), le second fait référence au discours de Roosevelt sur les quatre libertés prononcé en 1941. Ce dernier y exhortait le Congrès à débloquer des fonds pour faire la guerre à l'armée allemande, au nom d'un monde où règneraient la liberté d'expression, la liberté de croyance, la libération de la peur et de la misère. Le connecteur « et » qui sépare les deux énoncés est un connecteur temporel, puisqu'il met en relation deux événements contemporains de l'histoire de la guerre. Passons maintenant à l'observation génétique du préambule. Ci-dessous, on trouvera un tableau reprenant les différentes versions qu'a connu ce paragraphe au cours des discussions, avant la version finale adoptée par l'Assemblée Générale :

\begin{tabular}{|c|c|c|}
\hline $\begin{array}{l}\text { Première version par René } \\
\text { Cassin }(1) \\
(\mathrm{E} / \mathrm{CN} .4 / 21, \mathrm{p} .44)\end{array}$ & $\begin{array}{l}\text { Deuxième version, amendée } \\
\text { par la Comité de Rédaction } \\
(1 \text { ere session) }(2) \\
(\mathrm{E} / \mathrm{CN} .4 / 21, \text { p. } 54)\end{array}$ & $\begin{array}{l}\text { Troisième version, version amendée } \\
\text { par le comité de rédaction ( } 3 \text { e } \\
\text { session) }(3) \\
(\mathrm{E} / \mathrm{CN} .4 / 21, \mathrm{p} .67)\end{array}$ \\
\hline
\end{tabular}




\begin{tabular}{|l|l|l|l|}
\hline $\begin{array}{l}\text { Considérant que } \\
\text { l'ignorance et le mépris }\end{array}$ & $\begin{array}{l}\text { Considérant que l'ignorance et } \\
\text { le mépris des droits de }\end{array}$ & $\begin{array}{l}\text { Considérant que la méconnaissance } \\
\text { et le mépris des droits de l'homme }\end{array}$ \\
des droits de l'homme ont & l'homme ont été une des & ont conduit, dans la période qui a \\
été une des causes les plus & causes les plus importantes & précédé la Deuxième Guerre \\
importantes & des & des souffrances de l'humanité & Mondiale et durant cette guerre, à \\
souffrances de l'humanité, & et des menaces et actes de & des actes de barbarie révoltants pour \\
et, en particulier, des & barbarie qui ont fait outrage à & la conscience de l'humanité et qu'il \\
massacres qui ont souillé & la conscience humaine avant & est bien apparu qu'un des enjeux \\
la terre au cours de deux & et spécialement pendant la & suprêmes du conflit était les libertés \\
guerres mondiales & Deuxième Guerre Mondiale & fondamentales de l'homme \\
\hline
\end{tabular}

Comme on peut s'en apercevoir en comparant les trois versions, les rédacteurs ont progressivement restreint le cadre historique de référence à une période couvrant uniquement la Deuxième Guerre Mondiale. La première version renvoie aux deux guerres mondiales, la seconde accorde plus d'importance à la Deuxième Guerre Mondiale qu'à la Première, puisque la mention de la guerre 1914-18 disparaît. La troisième version, quant à elle, ne fait allusion qu'à la période entourant la Deuxième Guerre Mondiale - comme en atteste le second membre de l'énoncé qui fait allusion à un seul «conflit », anaphorique de l'expression «Deuxième Guerre Mondiale ». Dans (3), on insiste d'ailleurs sur le fait que la Deuxième Guerre Mondiale est la cause de la proclamation des droits de l'homme, en affirmant que les «libertés fondamentales » étaient l'un des enjeux suprêmes du conflit. Par ailleurs, les actes de barbarie qui provoquent la "révolte " ou l' "outrage " de la conscience de l'humanité désignent directement les actes perpétrés par les Nazis et plus spécifiquement le crime de génocide. Dans une résolution, les Nations Unies définissent d'ailleurs le génocide comme un crime qui « bouleverse la conscience humaine ${ }^{16} \mathrm{~A}$ la lecture des nombreux débats, on peut aussi que les «actes barbares » désignent de façon paradigmatique le génocide ${ }^{17}$.

Si les actes barbares renvoient aux actes perpétrés par les Nazis, le «mépris et la méconnaissance ${ }^{18}$ des droits de l'homme" doivent, logiquement, viser l'attitude adoptée par les Nazis face aux droits de l'homme, qui deviendrait de ce fait la définition même du nazisme. Une proposition d'amendement de la proposition (2), émise par la France, vient confirmer ce qui était à l'esprit des rédacteurs, en traçant un lien d'identité explicite entre « ignorance et mépris des droits de l'homme » à " nazisme et racisme » :

Considérant que l'ignorance et le mépris des droits de l'homme sont une des causes essentielles des souffrances de l'humanité ; que, spécialement dans la période qui a précédé la Deuxième Guerre Mondiale et durant cette guerre, le nazisme et le racisme ont été à l'origine d'actes de barbarie innombrables et révoltants pour la conscience humaine ${ }^{19}$.

En définissant dans l'alinéa 2 «nazisme » par « mépris et méconnaissance des droits de l'homme ", les rédacteurs portent un jugement de valeur sur le terme «nazisme » qui modifie de facto l'extension sémantique de «nazisme». Or, crucialement, ce jugement modifie en retour la dénotation de « droits de l'homme ».

Poursuivons notre raisonnement. Dans la version amendée par l'Assemblée Générale, deux modifications ont été effectuées. En premier lieu, tous les éléments qui rendaient l'énoncé explicitement spécifique à la Deuxième Guerre Mondiale ont été supprimés. Le 
motif invoqué est la crainte que la Deuxième Guerre Mondiale puisse être un jour oubliée et donc que la Déclaration apparaisse sans objet ${ }^{20}$. Or cet effacement des éléments explicites ne change à l'évidence rien aux représentations mentales véhiculées par la Déclaration, d'autant plus que les expressions implicites faisant référence au génocide sont restées en place. En second lieu, le deuxième membre de l'énoncé a été remplacé par une énumération des valeurs mises en avant par Roosevelt dans son discours.

\subsubsection{La notion d'« humanité »}

29

Intéressons-nous maintenant à la notion d'humanité telle que définie par la DUDH. Celle-ci joue ici un double rôle: elle confère à l'émotion de communion dans l'indignation le statut de fondement des droits de l'homme et en même temps, elle permet d'affirmer une opposition commune aux théories raciales et discriminatoires (qu'exprime la conscience révoltée de l'humanité, et souligné à nouveau dans l'article 1: «Ils [tous les hommes] sont doués de raison et de conscience et doivent agir les uns avec les autres dans un esprit de fraternité »). Toutes ces occurrences du terme d'humanité renvoient à la même définition, c'est-à-dire à une humanité victime de ou révoltée par les actes barbares. C'est donc une nouvelle vision de l'homme qui est ainsi explicitée par le texte, laquelle exprime de facto les critères d'inclusion et les critères d'exclusion à la communauté humaine. Ainsi, bien que les critères d'appartenance à l'humanité ne soient pas explicitement exprimés, on peut les déduire de l'emploi du terme dans le texte. Ainsi, les Nazis, ceux qui n'éprouveraient pas de compassion, ou encore ceux qui ne seraient pas révoltés par les actes barbares ne font pas partie de la communauté humaine telle qu'elle est définie par la Charte. Il suffit pour s'en convaincre de voir l'effet rhétorique que produit, dans le texte de la Charte, la substitution des occurrences de "humanité » et "êtres humains" par des sympathisants notoires du nazisme :

Considérant que la méconnaissance et le mépris des droits de l'homme ont conduit à des actes de barbarie qui révoltent la conscience de *Pétain, *Barbie et que l'avènement d'un monde où les *Pétain, *Barbie seront libres de parler et de croire, libérés de la terreur et de la misère, a été proclamé comme la plus haute aspiration de l'homme.

L'homme de la Déclaration Universelle est donc un homme qui a connaissance des actes perpétrés par les Nazis pendant la guerre, qui en est révolté et qui a un sentiment de compassion envers les victimes. Les droits de l'homme, dans leur version de 1948, sont les droits de ces hommes-là. Ils sont supposés donner à ces hommes les conditions politiques nécessaires à une vie de paix sans revivre les souffrances qui leur ont été infligées. A partir de cet arrière-plan topique se développera toute une rhétorique qui actualisera ces valeurs dans le monde politique de l'après Deuxième Guerre Mondiale en les considérant comme les fondements - nécessairement indiscutables - de ce nouveau monde ainsi déclaré. Les préambules qui précèdent la Déclaration proprement dite, sont une matrice rhétorique au niveau des contenus, parce qu'ils posent les piliers éthico-politiques qui vont déterminer ensuite la rhétorique. Ils déterminent qui fait partie de la communauté et qui hors de celle-ci, c'est-à-dire qui est atopos. Ils déterminent aussi les valeurs et les types de raisonnement qui vont être utilisés pour prendre les décisions politiques et judiciaires. Les principes argumentatifs à l'œuvre de ce nouveau monde commun consisteront, en gros, à juger de la qualité d'une décision dans sa capacité à s'opposer à un retour possible d'un régime nazi ${ }^{21}$. 


\section{3. L'articulation des alinéas 1 et 2}

(1), on l'a vu, exprime sous la forme d'une proposition générale des valeurs nouvelles qui reflètent l'opposition à l'idéologie nazie. Le considérant (2) exprime les valeurs au sein d'un récit historique contextualisé. On peut conclure de l'analyse qui précède que les deux énoncés expriment en fait la même chose, mais différemment. Les valeurs contenues dans (1) sont l'absolutisation de l'inverse des valeurs nazies exprimées dans (2). La reconnaissance des droits égaux et inaliénables et de la dignité de l'homme (C1) est la réponse au mépris des droits égaux et inaliénables (C2). On s'aperçoit en effet que (1) exprime sous une forme abstraite et générale l'inverse de ce que (2) raconte. Les valeurs de «dignité ", de "droits égaux et inaliénables » et de « liberté, paix, justice " sont absolutisées mais remplies d'un contenu mémoriel qui désigne spécifiquement l'histoire de la Deuxième Guerre Mondiale. Cet arrière-plan topique est un miroir du nazisme dont la fonction est de contrer l'avènement d'un monde nazi. Le monde de liberté, de paix et de justice idéalisé par les rédacteurs est celui où l'on aura la certitude que les actes barbares ne pourront plus se reproduire.

Pour terminer, mettons en lumière un problème concernant l'acceptabilité du préambule tel qu'il est exprimé. On l'a vu, les deux premiers "considérants" expriment la même chose, mais ont un statut différent. Dans le cas de (1), il s'agit d'une proposition générale qui exprime la réaction à (2). (2) exprime les raisons pour lesquelles ce constat a été élaboré. De ce fait, (2) justifie et explique comment on est parvenu à (1), ce qui est problématique au plan logique. En effet, (2) est, dans le texte, consécutif à (1). Cela rend l'articulation des deux énoncés difficilement acceptable, lorsqu'on les lit ensemble. En effet, les propriétés pragmatiques du connecteur «considérant que " qui précède les alinéas imposent que les énoncés se suivent de manière logique. En d'autres termes, le « considérant que » impose une chronologie de raisonnement qui organise l'ordre dans lequel les énoncés vont être placés. On peut tester cette hypothèse en ajoutant le connecteur «et, de ce fait» entre deux " considérants ", ce qui vérifie qu'il y a un lien, soit logique, soit chronologique entre les énoncés. En effet, dans le préambule, un énoncé entraîne souvent le suivant par un lien causal. Ainsi, on constate, en ajoutant le connecteur "et, de ce fait», que le préambule est tout à fait acceptable, des énoncés (2) à (8) alors que ce n'est pas le cas pour les énoncés (1) et (2) :

Considérant que la méconnaissance et le mépris des droits de l'homme ont conduit à des actes de barbarie qui révoltent la conscience de l'humanité et que l'avènement d'un monde où les êtres humains seront libres de parler et de croire, libérés de la terreur et de la misère, a été proclamé comme la plus haute aspiration de l'homme. $E t$, de ce fait, considérant qu'il est essentiel que les droits de l'homme soient protégés par un régime de droit pour que l'homme ne soit pas contraint, en suprême recours, à la révolte contre la tyrannie et l'oppression.

$E t$, de ce fait, considérant qu'il est essentiel d'encourager le développement de relations amicales entre nations. [...]

Cette version est acceptable, alors que la suivante non :

Considérant que la reconnaissance de la dignité inhérente à tous les membres de la famille humaine et de leurs droits égaux et inaliénables constitue le fondement de la liberté, de la justice et de la paix dans le monde.

$E t$, de ce fait, considérant que la méconnaissance et le mépris des droits de l'homme 
ont conduit à des actes de barbarie qui révoltent la conscience de l'humanité et que l'avènement d'un monde où les hommes seront libres de parler et de croire, libérés de la terreur et de la misère, a été proclamé comme la plus haute aspiration de l'homme.

De fait, inverser l'ordre des énoncés rend la consécution plus acceptable :

Considérant que la méconnaissance et le mépris des droits de l'homme ont conduit à des actes de barbarie qui révoltent la conscience de l'humanité et que l'avènement d'un monde où les hommes seront libres de parler et de croire, libérés de la terreur et de la misère, a été proclamé comme la plus haute aspiration de l'homme.

Et de ce fait, considérant que la reconnaissance de la dignité inhérente à tous les membres de la famille humaine et de leurs droits égaux et inaliénables constitue le fondement de la liberté, de la justice et de la paix dans le monde.

Pourquoi ce problème dans le préambule de la DUDH ? Sans doute les rédacteurs ont-ils passé plusieurs heures à organiser les énoncés d'une manière cohérente, mais on ne trouve aucune discussion sur un problème potentiel dans l'ordre de (1) et (2). La réponse se trouve dans les comptes rendus de rédaction du texte: à l'origine, les énoncés des "considérants» (1) et (2) appartenaient à deux propositions de préambules différentes que l'on a réunis dans un seul et même préambule. (1) provenait d'une proposition américaine qui se présentait comme suit:

CONSIDERANT que la reconnaissance de la dignité de la personne humaine et de l'égalité des droits pour tous est le fondement de la liberté, de la paix et de la justice dans le monde,

CONSIDERANT que les Etats Membres des Nations Unies, en proclamant à nouveau qu'ils reconnaissent cette dignité et ces droits, ont déclaré leur dessein de favoriser et d'encourager le respect des droits de l'homme et des libertés fondamentales

L'ASSEMBLEE GENERALE recommande la déclaration suivante comme représentant la mesure commune dans laquelle les Nations Unies s'acquitteront de l'obligation qui leur incombe de favoriser le respect des droits de l'homme et des libertés fondamentales pour tous, sans distinction de race, de sexe, de langue ou de religion.

Tandis que (2) provenait d'une version préliminaire proposée par la France que voici :

Nous, Peuples des Nations Unies,

1. CONSIDERANT que l'ignorance et le mépris des droits de l'homme sont une des causes essentielles des souffrances de l'humanité, des menaces et actes de barbarie qui ont fait outrage à la conscience humaine avant et spécialement pendant la dernière guerre mondiale.

2. Qu'il ne peut y avoir de paix que dans le respect des droits et des libertés de l'homme ; que le respect de ces droits et de ces libertés ne peut être assuré à tous que par l'abolition de la guerre et des menaces de guerre.

3. Que l'établissement d'un régime où les êtres humains, libres de parler et de croire, seront mis à l'abri, de la terreur et de la misère, a été proclamé comme l'enjeu suprême de la récente lutte [...]

EN CONSEQUENCE, nous avons adopté la déclaration suivante : [...]

On comprend dès lors ce qui s'est produit: le préambule français étant considéré comme trop contextuel et ancré dans son histoire puisque tous les alinéas font référence à la Deuxième Guerre Mondiale, les rédacteurs ont souhaité lui ajouter, à la tête $\mathrm{du}$ texte, une formule générale qui permette de rendre les valeurs plus universelles. Malik, l'un des rédacteurs du préambule, s'est même félicité de la nouvelle organisation du préambule en affirmant qu'au paragraphe 1 , on exprimait un principe "absolu, d'ordre général » tandis qu'au paragraphe 2, on affirmait que ce principe « avait été violé ». Or il semble que le statut des énoncés soit plutôt, comme on l'a dit, l'idée qu'on affirme un principe absolu avant d'affirmer comment ce principe a émergé. 
Mais dans le chef des rédacteurs, la volonté de faire face aux deux exigences, identification épidictique et généralisation à visée universelle a abouti à cette structure qui permet de faire «comme si » les valeurs existaient avant même que l'expérience nous ait poussé à devoir les penser. En arrière-plan de cette contradiction se trouve peut-être les prémisses du débat classique sur l'universalité des droits de l'homme, puisque les deux considérants témoignent d'une hésitation originelle sur le sens des valeurs : préexistantes à l'existence de l'homme ou élaborées en contexte après avoir constaté la catastrophe de la Deuxième Guerre Mondiale.

\section{Conclusion}

Les valeurs peuvent être exprimées de deux façons différentes, lesquelles traduisent deux fonctions différentes de la Déclaration. En effet, alors que le contenu représentationnel des droits de l'homme est clairement contextualisé quand la Charte doit remplir sa fonction épidictique, on fera «comme-si » (voir Danblon 2002) il ne l'était pas dans sa fonction délibérative et on le considérera comme déconnecté du contexte particulier et faisant partie d'un univers général. Par conséquent, les droits de l'homme seront en permanence difficiles à définir et cette difficulté subsistera dans l'arrière-plan topique: ne voyons-nous pas tous les jours s'affronter des philosophes ou des politologues sur le sens des droits de l'homme, les uns définissant les droits de l'homme par la définition de leur violation (ce qui serait plutôt une position ou l'on rattache les droits de l'homme au contexte historique de la DUDH), les autres cherchant à donner aux valeurs de droits de l'homme une existence indépendante et éternelle, en arguant que la DUDH ne fait qu'exprimer des revendications ayant existé à toutes les époques de l'histoire.

L'arrière-plan topique qu'exprime la DUDH est articulé à une période déterminée de l'histoire. On peut observer aujourd'hui les conséquences de ce fait : paradoxalement, les sociétés européennes ont besoin d'une représentation du régime nazi pour que leur vision du monde ait du sens. En effet, pour pouvoir définir les droits de l'homme en termes de représentations collectives, les individus vont aller constamment puiser dans les contre-exemples que fournissent le régime nazi pour redéfinir le sens et les limites des droits de l'homme. A l'heure actuelle, où le souvenir de la Deuxième Guerre Mondiale s'estompe progressivement au fur et à mesure que cette période s'éloigne, on peut s'interroger sur le devenir d'une telle vision du monde, et se demander comment le monde commun va faire face à cet effacement programmé de ses fondements.

\section{BIBLIOGRAPHIE}

L'ensemble des archives sur la rédaction de la Déclaration Universelle des Droits de l'Homme peut être consulté sur le site http://www.unog.ch/library.

Agi, Marc. 1979. René Cassin, Fantassin des droits de l'Homme (Paris : Plon) 
Aristote. 1990. Rhétorique (Paris : Les Belles Lettres)

Aristote. 1967. Topiques. Tome I (Paris : Les Belles Lettres)

Angenot, Marc. 2008. Dialogue de sourds : Traité de Rhétorique antilogique (Paris : Mille et Une Nuits)

Danblon, Emmanuelle. 2002. Rhétorique et rationalité. Essai sur l'émergence de la critique et de la persuasion (Bruxelles : Editions de l'Université de Bruxelles)

Danblon, Emmanuelle. 2004. Argumenter en démocratie (Bruxelles : Editions Labor)

De Smet, François. 2001. Les droits de l'homme : origines et aléas d'une idéologie moderne (Paris :

Editions du Cerf)

de Jonge, Emmanuel. 2008a. « Le préambule des chartes et des déclarations ». Danblon, Emmanuelle et al. (éds). Argumentation et narration (Bruxelles : Editions de l'Université Libre de Bruxelles), 99-115

de Jonge, Emmanuel. 2008b. « Pertinence de l'utilisation du modèle de Toulmin dans l'analyse de corpus », Argumentation et Analyse du Discours 1, «L'analyse du discours au prisme de l'argumentation » [mis en ligne le 18 septembre 2008. URL : http://aad.revues.org/ index251.html]

Marshall,Peter. 2001. « Smuts And The Preamble To The UN Charter », The Round Table $90: 358$, 55-65

Morsink, Johannes. 2000. The Universal Declaration of Human Rights: Origins, Drafting and Intent (Philadelphia : University of Pennsylvania Press)

Perelman, Chaïm et Lucie Olbrechts-Tyteca. 1988 [1958]. Traité de l'argumentation (5éd.)

(Bruxelles : Éditions de l'Université de Bruxelles)

Schaeffer, Jean-Marie. 2008. La Fin de l'Exception Humaine (Paris : NRF)

verdoodt, Albert. 1964. Naissance et signification de la Déclaration Universelle des Droits de l'Homme (Louvain : E. Warny)

\section{NOTES}

1. Ainsi René Cassin, l'un des principaux rédacteurs de la Déclaration, affirmait dans un entretien : «Le mot "universelle" je l'ai improvisé en séance. [...] l'idée d'universalité s'est plus précisément accusée au fur et à mesure des travaux, sur le plan des droits. Au début, les nations occidentales concevaient une déclaration des droits de l'homme analogue à la déclaration de 89 ou à la déclaration américaine : le droit au bonheur, la liberté d'expression, de réunion etc. Mais il faut bien dire que beaucoup de nations voulaient qu'on parlât d'abord du droit à la vie. Moi j'ai mis dès le début dans mon premier article "droit à la vie, à la liberté, à la sûreté" [...] Quand j'ai fait proclamer que la déclaration serait universelle, j'ai voulu indiquer que chaque être humain était membre d'une société mondiale, et pas seulement sujet de son état et indirectement sujet des nations unies » (Agi 1979 : 355-356).

2. L'absence de tous ces éléments dans les Constitutions, qui sont des documents fort proches des déclarations au point de vue de la forme explique d'ailleurs en grande partie pourquoi cellesci sont très rarement considérées comme objets de communion épidictique pas plus que leur contenu n'est mentionné dans un discours à visée épidictique.

3. A/C.3/314/Rev.1; page 2. La proposition d'amendement a été rejetée. L'argument des PaysBas faisait valoir qu'une déclaration aussi importante que celle-ci devait nécessairement 
comporter une dimension métaphysique et faire mention d'un Etre Suprême auquel la majorité des peuples croyaient.

4. A/C.3/314/ Rev.1; page 4. La proposition d'amendement a été rejetée.

5. Le fait que cette version n'ait pas été choisie n'empêche pas aujourd'hui certains détracteurs de la DUDH de la dénoncer au motif qu'elle serait un dogme comme un autre.

6. Excepté une modification dans le texte anglais, sans effet sur le texte français, qui consistait à ajouter « of » devant les mots « the equal » : "Whereas recognition of the inherent dignity and of the equal and inalienable rights of all members of the human family is the foundation of freedom, justice and peace in the world" (E/CN.4/SR.75/ page 6).

7. M. Azkoul, E/CN.4/SR.75/ page 6.

8. M. Chang, E/CN.4/SR.75/ page 6 .

9. Auquel on doit ajouter le souvenir récent de la Première Guerre Mondiale dans la mémoire collective.

10. On y a ajouté le terme "inhérente» pour insister sur le caractère naturel et donc indiscutable de cette notion.

11. E/CN.4/95, page 6 ; c'est moi qui souligne. Traduit par « dégradant » en français.

12. M. Malik, E/CN.4/SR.21

13. E/CN.4/95; page 2

14. voir à ce sujet Marshall 2001.

15. L'alinéa se lit comme suit en anglais "Whereas disregard and contempt for human rights have resulted in barbarous acts which have outraged the conscience of mankind, and the advent of a world in which human beings shall enjoy freedom of speech and belief and freedom from fear and want has been proclaimed as the highest aspiration of the common people."

16. Résolution de l'Assemblée Générale des Nations Unies, A/RES/96(I), page 188-189

17. A titre informatif, on peut souligner le fait que la plupart des membres du Comité de Rédaction de la Déclaration Universelle des Droits de l'Homme faisaient également partie du Comité du Génocide, chargé de rédiger une convention sur le génocide. Certaines sessions ont même eu lieu simultanément.

18. Soulignons la substitution du terme "ignorance " pour le terme "méconnaissance ». Les rédacteurs ont estimé qu'il fallait éviter que les Nazis ne soient excusés parce qu'on aurait considéré qu'ils ne savaient pas qu'ils violaient les droits de l'homme. C'est pour cette raison que les rédacteurs ont modifié le terme. La même substitution a été opérée dans le texte anglais où " disregard », qui véhicule l'idée d'une intention de mal faire, a été préféré à " ignorance ».

19. A/C.3/339, page 1

20. A/C.3/352, page 751

21. Et ce alors même que dans l'esprit, le monde commun est proclamé comme universel pour la première fois.

\section{RÉSUMÉS}

Dans cet article, je réalise une analyse de la Déclaration Universelle des Droits de l'Homme dans une perspective rhétorique et génétique. A l'aide des comptes rendus des débats préliminaires du Comité de Rédaction de la Déclaration, je montre que le texte a pour arrière-plan la volonté de fonder une vision du monde nouvelle, dans laquelle tout serait mis en œuvre d'un point de vue 
juridique, éthique, philosophique et psychologique pour que les actes subis par les populations européennes durant la Seconde Guerre Mondiale ne puissent plus se reproduire. Pour ce faire, j'analyse la question de l'expression linguistique des valeurs dans les deux premiers alinéas du préambule de la Déclaration.

In this contribution, I analyze the text of the Universal Declaration of Human Rights from a rhetorical and genetic perspective. Starting from the summary reports of the Declaration Drafting Committee, I try to show that what lies at the background of the text is the feeling that the sufferings of WWII never can be reproduced. The drafters thus founded a new "vision of the world" that has shaped our values since then. I concentrate specifically on the first two lines of the preamble of the Declaration in order to analyze how the values are expressed linguistically in these paragraphs.

INDEX

Mots-clés : déclaration universelle, démocratie, discours des droits de l'homme, topique, vision du monde

Keywords : democracy, human rights discourse, topic, universal declaration, vision of the world

\section{AUTEUR}

EMMANUEL DE JONGE

ULB, GRAL 